Health \& Medicine | Yoshitsugu Aoki

\section{Stem cells could offer hope for muscular dystrophy}

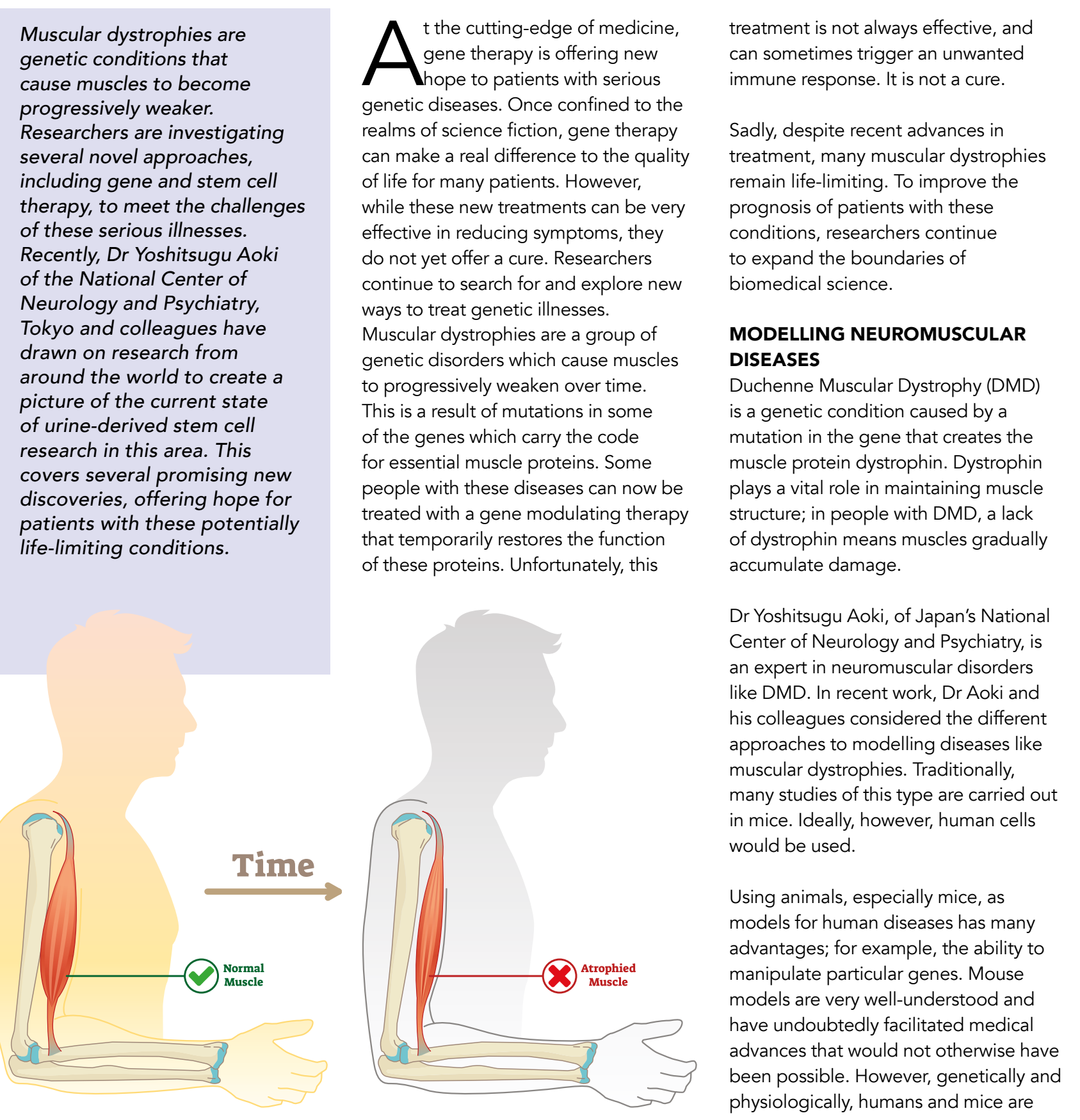

very different. Comparing the ways in which both diseases and treatn is challenging

roblem could be to use human cells for disease modelling. Dr Aoki and his colleagues have recently considered promising sources of cells for this purpose, including urine-derived cells (UDCs). Unlike other possible sources, such as skin, muscle or blood samples, cells can be obtained from urine non-invasively and with no risk of harm to the patient. They can also be collected repeatedly (and cheaply) from the same patient.

Urine-derived cells have already shown their potential in modelling numerous Aoki and his team developed a new, Aoki and his team developed a new, in UDCs. The cells used originate in the kidneys and urinary tract and possess the properties of stem cells; that is, they are able to develop into several different types of cells, with different functions.

The team used UDCs in two ways. First, they successfully encouraged the UDCs to develop into healthy myotubes (muscle fibre cells). This was achieved by manipulating a gene called MYOD1, which plays an essential role in muscle development. In this work, the team used MTODI to effectively reIn created in vitro (outside the body) with this method could be used to investigate the precise nature of the disease in each

Second, Dr Aoki used the UDCs to explore an exciting genetic technique called "exon-skipping." In exonskipping, cells are prompted to "skip" the mutated part of a gene when making a protein. This is done by building a short piece of nucleic acid (the substance from which DNA is made) to bind to the mutation, which causes that part of the genetic code to be skipped. The result is a protein that but is still functio foun is currently attraling a grostiping

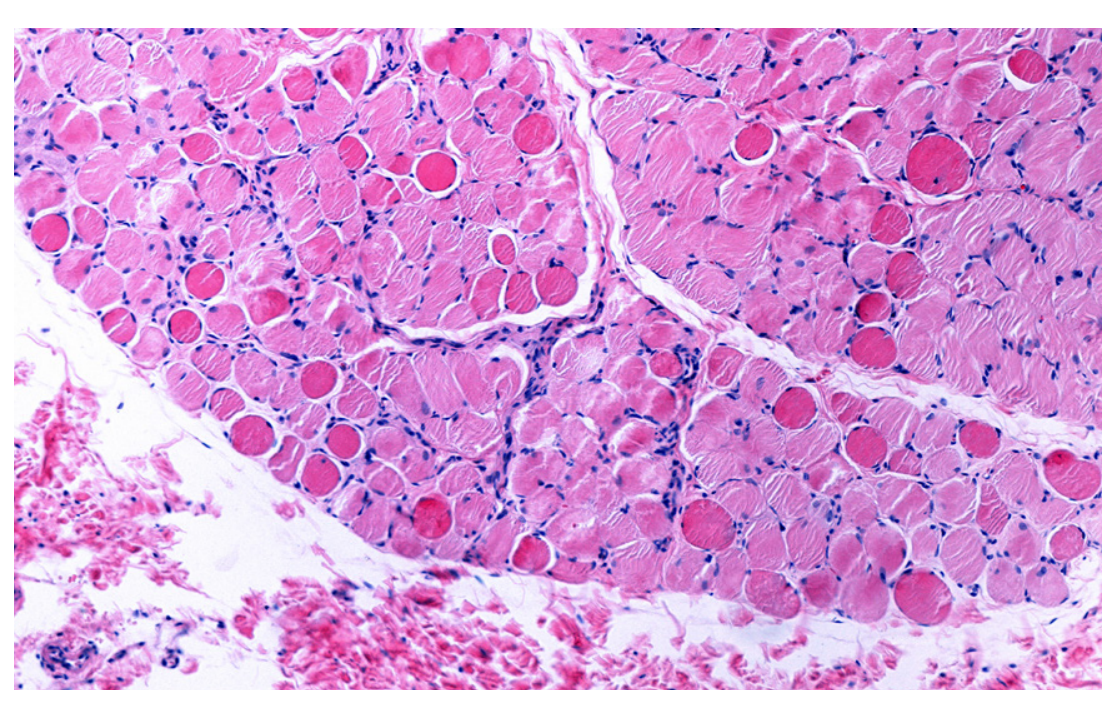
A key pathologic change of Duchenne muscular dystrophy is myonecrosis. At an early phase,
necrotic fibres appear swollen,

research interest as a potential treatment for DMD, as it could allow cells to create functional dystrophin.

The team were able to successfully demonstrate exon-skiping in UDCs from DMD patients with different types of mutation. They also discovered that their UDC model can be used to identify clinical trials, whether the exon skipping in vitro is as effective when used in vivo.

\section{ROM MODELLING TO}

STEM CELL TREATMENT

In another strand of work, Dr Aok a review into the newest, most advanced treatments for muscular

Researchers have identified a number of different stem cell types which could be adapted to treat muscular dystrophies.

skip the mutated part of the gene. This could allow each DMD patient to be

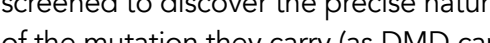
result from more than one muthion) As a result, a personalised exon-skipping treatment could be developed.

While the techniques described by $\mathrm{Dr}$ Aoki and his colleagues require further refinement, the results of this research are extremely promising. An importan future step will be to test, through

dystrophies. In this, Dr Aoki and his colleagues had a particular focus on DMD and on one type of potentially stem cell therapy.

Normally, skeletal muscle (i.e. the muscles that are attached to bones) has a remarkable ability to heal itself after injury. Muscle stem cells, particularly a type called satellite cells, play a crucial role in the healing process. For this reason, researchers have targeted

Muscle development

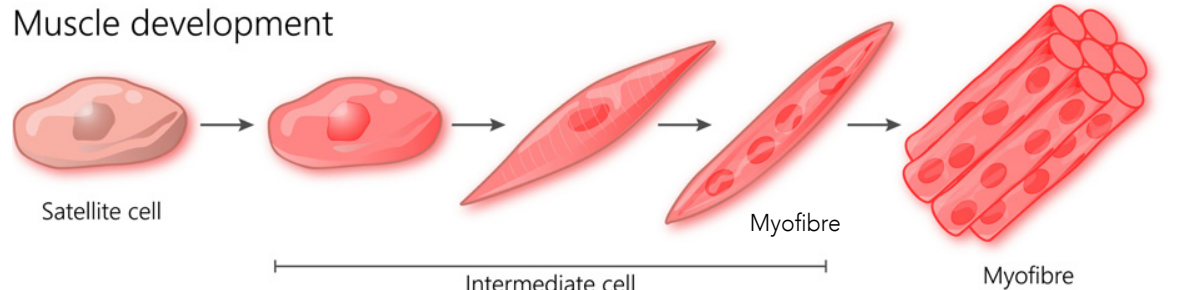

Muscle stem cells, particularly a type called satellite cells, play a crucial role in the healing process. 


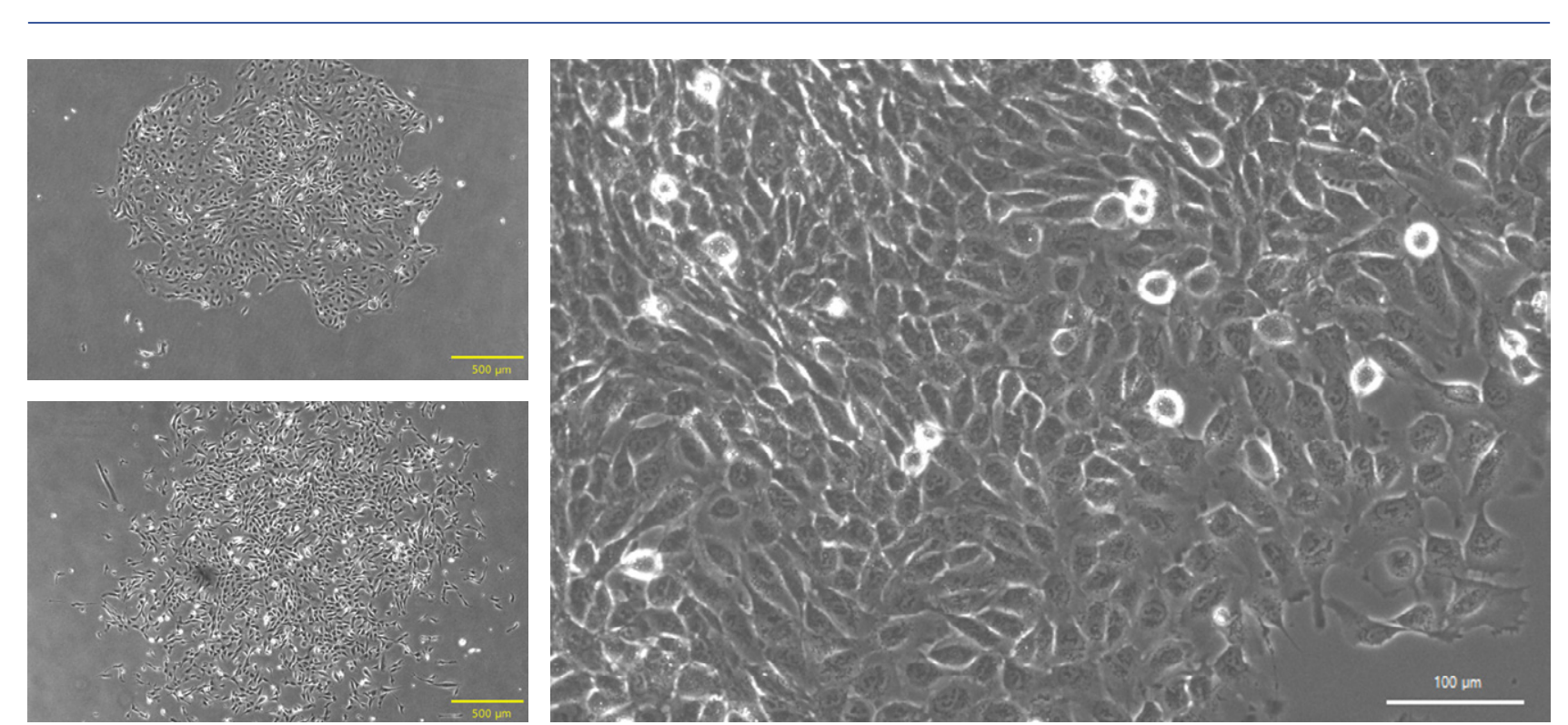

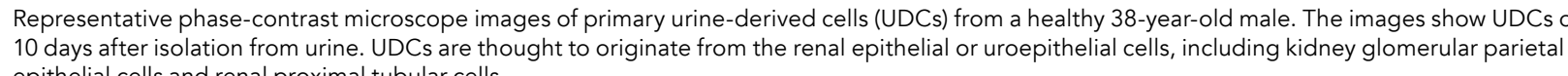

New genetic techniques are offering different ways to tackle the problems of gene mutations behind diseases like DMD.

satellite cells as a potential treatment route for muscular dystrophies. Studies using mice have produced promising results, with transplanted satellite cel leading to restored dystrophin and better muscle function. Research with DMD patients has begun, however, more work is needed before this can become a widely-used treatment

As with all types of transplant, there is be rsibility hat donor stem cells w solution would be to use the patient's own cells which have been collected genetically modified to "fix" the faulty dystrophin gene. However, the genetic modification process can render the stem cells less effective.

GROWING STEM CELLS

Another challenge is generating enoug stem cells for an effective treatment. In some muscular dystrophies (including DMD), most muscles in the body are affected, which means that treatment would require a very large number of modified stem cells. Stem cells, once collected, need to be expanded (i.e. grown in number) in vitro before
When stem cells are grown in vitro, their capacity for generating new muscle tends to reduce. Researchers have discovered that a very specific environment is needed in order for stem cells to function at their ful potential, both in vitro and in vivo, i.e. in the muscle. Understanding how environment affects muscle stem cells is

\section{SUPPORTING STEM CELLS} mimics their normal environment. This substance can contain other types of muscle cell, or biomaterials - manmade substances built to interact with biological systems.

Another approach is to actually create artificial muscles in vitro, using satellite cells and other muscle cells combined with extracellular matrix. Muscle tissue created in this way can partially restore the disease. crucial if this approach to treatmen

One strategy to help muscle stem cells to coat the cells with a substance that the function of damaged muscles. Like stem cells, it can also be used as a usefu
These methods hold great potential in repairing damaged muscles. However, applying these techniques as treatments for conditions like DMD, where entive become a reality.

\section{THE FUTURE OF MUSCULAR} DYSTROPHY RESEARCH AND TREATMENT

Stem cells are a valuable tool in muscular dystrophy research in two ways: by their role as a direct treatment for the stem cell transplantation may not only people with are dystrophin function in people with muscular dystrophies, but pation migh also rejurenate the cellular further progression of the disease. At the same time, new get tease. At like exon-skipping are offering different mutations behind diseases like DMD.

Ultimately, these innovative approaches - in disease modelling, in gene therapy and in stem cell treatment - seek to do one thing: improve the prospects of those living with serious genetic conditions like muscular dystrophies. The challenge taken on by Dr Aoki and other researchers, of developing combined stem cell transplantation medicine and precision exon skipping approaches for restoring tissue allow this goal to becom, may soon acting as models for the disease, and in condition. Recent work has shown that ways to tackle the problems of the gene

\section{Behind the Research}

\section{Dr Yoshitsugu Aoki}

E: tsugu56@ncnp.go.jp T: +81-42- 346-1720 F: +81-42- 346-1750 W: https://www.ncnp.go.jp/nin/quide/r_dna2/en/index.html

\section{Research Objectives}

Integrating molecular, pharmacologic, proteomic and genomic methods, Dr Aoki's research aims to elucidate herapy for fatal Duchenne muscular dystrophy and other neuromuscular diseases.

\section{Detail}

Yoshitsugu Aoki
4-1-1, Ogawa-higashi

odaira, Tokyo 187-8551, Japan

Bio

Droshitsugu Aoki was MRC postdoctoral researcher and then Senior Research Scientist, Faculty of 2015. He is currently Research Director and Consultan . National Center of Neurology and Psychiatry, Tokyo.

Funding

作 Disorders [number 2-6], and the Japan Agency for Medical Research and Develop Jant Lrant numbers 81 m0203069h0001.

\section{Collaborators}

Dr Hotake Takizawa at National Center of Neurology and Psychiatry (NCNP)

- Dr Norio Motohasin

- Prof. Matthew JA Wood University of Oxford - Prof. Toshifumi Yokota, University of Alberta

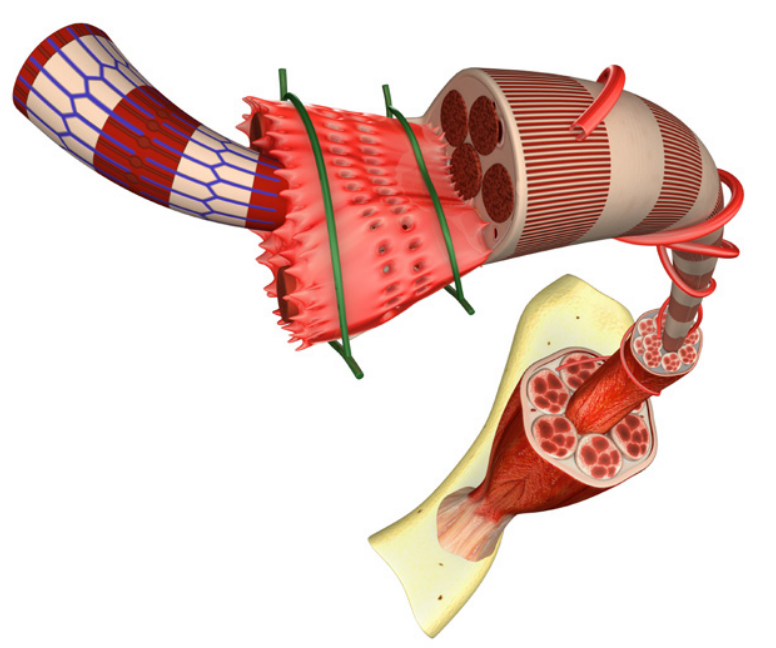

\section{References}

Takizawa, H, et al. 2019. Modelling Duchenne Muscular Dystrophy in MYOD1-converted Urine-Derived Cells $2020 \mathrm{Feb}$ 7:10(1):2462. doi:10.1038/s41598-020-59351-2

Motohashi, N, et al. 2019. Potential therapies using myogenic stem cells combined with bio-engineering approaches for treatment of muscular
8(1066) doi:10.3390/cells8091066

Sato, M, et al. 2019. Application of urine-derived stem cells to cellular modelling in neuromuscular and neurodegenerative diseases. Frontiers in Molecular Neuroscience 12(297) doi:10.3389/fnmol.2019.0029

\section{Personal Response}

How soon are we likely to see stem cell treatment become routine?

II Efficacy and safety, as well as quality, cost and speed of new medicine, are essential factors for the development
of stem cell therapies. To date, autologous transplantation medicine using cultured epidermal stem cells and chondrocytes is a common approach for skin and articular cartilage repair. In addition, allogeneic hematopoietic stem cell transplantation wh in incosuppressive agents hematologic malignancies.

Currently, stem cell therapy using Nobel-winning induced plurpotent stem cells (iPSC) or urine-derived cells (UDC) iPSC-derived retina cell transplant for agereled macular degeneration are ongoing in Japan and USA. PSSC and UDC could be used in the treatment of musculoskeletal, cardiovascular and autoimmune disorders, cancer, and drug to transform the healthcare industry, and the global stem cells market size may reach 11 billion USD by 2029. 